\title{
HIGH ENERGY LIMITS OF LAPLACE-TYPE AND DIRAC-TYPE EIGENFUNCTIONS AND FRAME FLOWS
}

\author{
DMITRY JAKOBSON AND ALEXANDER STROHMAIER
}

\begin{abstract}
We relate high-energy limits of Laplace-type and Diractype operators to frame flows on the corresponding manifolds, and show that the ergodicity of frame flows implies quantum ergodicity in an appropriate sense for those operators. Observables for the corresponding quantum systems are matrix-valued pseudodifferential operators and therefore the system remains non-commutative in the high-energy limit. We discuss to what extent the space of stationary high-energy states behaves classically.
\end{abstract}

\section{INTRODUCTION AND MAIN RESULTS}

If $X$ is an oriented closed Riemannian manifold and $\Delta$ the Laplace operator on $X$, then a complete orthonormal sequence of eigenfunctions $\phi_{j} \in L^{2}(X)$ with eigenvalues $\lambda_{j} \nearrow \infty$ is known to converge in the mean to the Liouville measure, in the sense that

$$
\lim _{N \rightarrow \infty} \frac{1}{N} \sum_{j \leq N}\left\langle\phi_{j}, A \phi_{j}\right\rangle=\int_{T_{1}^{*} X} \sigma_{A}(\xi) d L(\xi),
$$

for any zero order pseudodifferential operator $A$, where integration is with respect to the normalized Liouville measure on the unit cotangent bundle $T_{1}^{*} X$, and $\sigma_{A}$ is the principal symbol of $A$. In particular, $A$ might be a smooth function on $X$ and the above implies that the sequence

$$
\frac{1}{N} \sum_{j \leq N}\left|\phi_{j}(x)\right|^{2}
$$

Date: July 15, 2018.

2000 Mathematics Subject Classification. Primary: 81Q50 Secondary: 35P20, 37D30, 58J50, 81Q005.

Key words and phrases. Dirac operator, Hodge Laplacian, eigenfunction, frame flow, quantum ergodicity.

The first author was supported by NSERC, FQRNT and Dawson fellowship. 
converges to the normalized Riemannian measure in the weak topology of measures. In case the geodesic flow on $T_{1} X$ is ergodic it is known that the following stronger result holds.

$$
\lim _{N \rightarrow \infty} \frac{1}{N} \sum_{j \leq N}\left|\left\langle\phi_{j}, A \phi_{j}\right\rangle-\int_{T_{1}^{*} X} \sigma_{A}(\xi) d L(\xi)\right|=0 .
$$

This property is commonly referred to as quantum ergodicity and it is equivalent to the existence of a density-one-subsequence $\phi_{j}^{\prime}$ such that

$$
\lim _{j \rightarrow \infty}\left\langle\phi_{j}^{\prime}, A \phi_{j}^{\prime}\right\rangle=\int_{T_{1}^{*} X} \sigma_{A}(\xi) d L(\xi),
$$

for any zero order pseudodifferential operator $A$ (see Shn74, Shn93, CV85, Zel87]).

We show in this paper that the high energy behavior of the Dirac operator $D$ acting on spinors on a closed spin manifold $X$ is determined by the frame flow in the same manner, as the geodesic flow determines the high energy limit of the Laplace operator. If $F_{k} X$ is the bundle of oriented orthonormal $k$-frames in $T^{*} X$, then projection to the first vector makes $F_{k} X \rightarrow T_{1}^{*} X$ into a fiber bundle. In particular for $k=n$ this is the full frame bundle and $F X=F_{n} X$ is a principal fiber bundle over $T_{1}^{*} X$ with structure group $S O(n-1)$. Transporting covectors parallel along geodesics extends the Hamiltonian flow on $T_{1}^{*} X$ to a flow on $F_{k} X$. This is the so-called $k$-frame flow. In case $k=n$ we will refer to it simply as the frame flow. Of course ergodicity of the $k$-frame flow for any $k$ implies ergodicity of the geodesic flow, whereas the conclusion in the other direction is not always true (cf. section 2). Still there are many examples investigated in the literature when the frame flow is ergodic. Our first main result is, that quantum ergodicity holds for eigensections of the Dirac operator in case the frame flow is ergodic.

Theorem 1.1. Let $X$ be a closed Riemannian spin manifold of dimension $n \geq 3$ with Dirac operator $D$ acting on sections of the spinor bundle. Suppose that $\phi_{j} \in L^{2}(X ; S)$ is an orthonormal sequence of eigensections of $D$ with eigenvalues $\lambda_{k} \nearrow \infty$ such that the $\phi_{k}$ span $^{1}$ the positive energy subspace of $D$. Then, if the frame flow on $F X$ is ergodic, we have

$$
\lim _{N \rightarrow \infty} \frac{1}{N} \sum_{j \leq N}\left|\left\langle\phi_{j}, A \phi_{j}\right\rangle-\frac{1}{2^{\left[\frac{n}{2}\right]}} \int_{T_{1}^{*} X} \operatorname{Tr}\left((1+\gamma(\xi)) \sigma_{A}(\xi)\right) d L(\xi)\right|=0,
$$

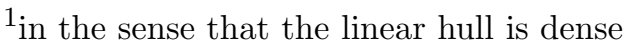


for all $A \in \Psi_{D^{\prime}}^{0}(X, S)$. Here $\gamma(\xi)$ denotes the operator of Clifford multiplication with $\xi$. In particular there is a density one subsequence $\phi_{j}^{\prime}$ such that

$$
\left\langle\phi_{j}^{\prime}, A \phi_{j}^{\prime}\right\rangle \rightarrow \frac{1}{2^{\left[\frac{n}{2}\right]}} \int_{T_{1}^{*} X} \operatorname{Tr}\left((1+\gamma(\xi)) \sigma_{A}(\xi)\right) d L(\xi) .
$$

A similar statement holds for the negative energy subspace.

Another result is that the $(2 \min (p, n-p))$-frame flow determines the high energy behavior of the Laplace-Beltrami operator $\Delta_{p}$ acting on the space $C^{\infty}\left(X, \Lambda_{\mathbb{C}}^{p} X\right)$ of complex-valued $p$-forms. Note that the Hodge decomposition implies that there are three invariant subspaces for $\Delta_{p}$, namely the closures of $d C^{\infty}\left(X, \Lambda_{\mathbb{C}}^{p-1} X\right), \delta C^{\infty}\left(X, \Lambda_{\mathbb{C}}^{p+1} X\right)$ and the finite dimensional space of harmonic forms The latter subspace plays no role for the high energy behavior. The eigenspaces of the first subspace consist of exterior derivatives of $p-1$ eigenforms their high energy behavior is therefore determined by the high energy behavior of $\Delta_{p-1}$. In particular the high energy behavior of $\Delta_{1}$ restricted to $d C^{\infty}(X)$ is controlled by the geodesic flow. We therefore look at the second subspace only. Note that any coclosed form which is a nonzero eigenvalue to $\Delta_{p}$ is coexact. Hence, to investigate the high energy behavior of $\Delta_{p}$ we have to look at the system

$$
\begin{gathered}
\Delta_{p} \phi_{j}=\lambda_{j} \phi_{j}, \\
\delta \phi_{j}=0 .
\end{gathered}
$$

In case $p=1$ such systems appear in physics if one investigates electromagnetic fields (Maxwell's equations) or the Proca equation for spin 1 particles. The restriction to the coclosed forms corresponds to a gauge condition which restricts to the transversal subspaces. Our result is, that this system is quantum ergodic, if the $2 \min (p, n-p)$-frame flow is ergodic.

Theorem 1.2. Let $X$ be an oriented closed Riemannian manifold of dimension $n \geq 3$ and let $0<p<n$. Suppose that $\phi_{k}$ is an orthonormal sequence of eigen-p-forms satisfying

$$
\begin{gathered}
\Delta_{p} \phi_{k}=\lambda_{k} \phi_{k}, \\
\delta \phi_{k}=0,
\end{gathered}
$$

such that the $\phi_{k}$ span $\operatorname{ker}(\delta)$ and with $\lambda_{k} \nearrow \infty$. Suppose that $p \neq$ $\frac{n-1}{2}$. Then, if the $(2 \min (p, n-p))$-frame flow is ergodic, the system is 
quantum ergodic in the sense that

$$
\lim _{N \rightarrow \infty} \frac{1}{N} \sum_{k \leq N}\left|\left\langle\phi_{k}, A \phi_{k}\right\rangle-\omega_{t}\left(\sigma_{A}\right)\right|=0,
$$

for all $A \in \Psi \mathrm{DO}_{c l}^{0}\left(X ; \Lambda_{\mathbb{C}}^{p} X\right)$. In particular there is a density one subsequence $\phi_{k}^{\prime}$ such that

$$
\lim _{k \rightarrow \infty}\left\langle\phi_{k}^{\prime}, A \phi_{k}^{\prime}\right\rangle=\omega_{t}\left(\sigma_{A}\right), \quad \text { for all } A \in \Psi \mathrm{DO}_{c l}^{0}\left(X ; \Lambda_{\mathbb{C}}^{p} X\right) .
$$

Here $\omega_{t}$ is a state on the $C^{*}$-algebra of continuous $\operatorname{End}\left(\Lambda_{\mathbb{C}}^{p} X\right)$-valued functions on $T_{1}^{*} X$ which is defined by

$$
\omega_{t}(a):=\left(\begin{array}{c}
n-1 \\
p
\end{array}\right)^{-1} \int_{T_{1}^{*} X} \operatorname{Tr}\left(i(\xi) i^{*}(\xi) a(\xi)\right) d L(\xi),
$$

where $i(\xi)$ is the operator of interior multiplication with $\xi$, and the adjoint $i^{*}(\xi)$ is the operator of exterior multiplication with $\xi$.

Note that the system

$$
\begin{gathered}
\Delta_{k} \phi_{j}=\lambda_{j} \phi_{j}, \\
d \phi_{j}=0 .
\end{gathered}
$$

is equivalent to our system with $p=n-k$ via the Hodge star operator.

The restriction $p \neq \frac{n-1}{\frac{2}{2}}$ is necessary since if $p=\frac{n-1}{2}$ the operator $\mathrm{i}^{p+1} \delta *$ leaves the space $\overline{\operatorname{Rg}(\delta)}$ invariant and commutes with $\Delta_{p}$ (* is the Hodge star operator). In this case our result is

Theorem 1.3. Let $X$ be an oriented closed Riemannian manifold of odd dimension $n \geq 3$. Let $p=\frac{n-1}{2}$ and suppose that $\phi_{k}$ is an orthonormal sequence of eigen-p-forms satisfying

$$
\begin{aligned}
\Delta_{p} \phi_{k} & =\lambda_{k} \phi_{k}, \\
\delta \phi_{k} & =0, \\
\mathrm{i}^{p+1} \delta * \phi_{k} & = \pm \sqrt{\lambda_{k}} \phi_{k}
\end{aligned}
$$

such that the $\phi_{k}$ span $\overline{\operatorname{Ran}\left(\delta \pm \mathrm{i}^{p+1} \Delta_{p}^{-1 / 2} \delta * \delta\right)}$ and with $\lambda_{k} \nearrow \infty$. Then, if the $(n-1)$-frame flow is ergodic, the system is quantum ergodic in the sense that

$$
\lim _{N \rightarrow \infty} \frac{1}{N} \sum_{k \leq N}\left|\left\langle\phi_{k}, A \phi_{k}\right\rangle-\omega_{ \pm}\left(\sigma_{A}\right)\right|=0
$$

for all $A \in \Psi \mathrm{DO}_{c l}^{0}\left(X ; \Lambda_{\mathbb{C}}^{p} X\right)$. In particular there is a density one subsequence $\phi_{k}^{\prime}$ such that

$$
\lim _{k \rightarrow \infty}\left\langle\phi_{k}^{\prime}, A \phi_{k}^{\prime}\right\rangle=\omega_{ \pm}\left(\sigma_{A}\right), \quad \text { for all } A \in \Psi \mathrm{DO}_{c l}^{0}\left(X ; \Lambda_{\mathbb{C}}^{p} X\right) .
$$


Here the states $\omega_{ \pm}$are defined by

$$
\omega_{ \pm}(a):=\frac{(p !)^{2}}{(2 p) !} \int_{T_{1}^{*} X} \operatorname{Tr}\left(\left(1 \pm \mathrm{i}^{p} i(\xi) *\right) i(\xi) i^{*}(\xi) a(\xi)\right) d L(\xi) .
$$

As the example of Kähler manifolds (see section 21) show the above theorems do not hold if we assume ergodicity of the geodesic flow only.

Our analysis is based on a version of Egorov's theorem for matrix valued operators. A second order differential operator $P$ acting on sections of a vector bundle $E$ is said to be of Laplace type if $\sigma_{P}(\xi)=g(\xi, \xi) \mathrm{id}_{E}$, i.e. if in local coordinates it is of the form $P=-\sum_{i, k} g^{i k} \partial_{i} \partial_{k}+$ lower order terms. Examples are the LaplaceBeltrami operator $\Delta_{p}$ acting on $p$-forms or the square $D^{2}$ of the Dirac operator on a Riemannian spin manifold. For such operators the first order term (the subprincipal symbol) defines a connection $\nabla^{E}$ on the bundle $E$. We will prove a Egorov theorem for matrix-valued pseudodifferential operators acting on sections of $E$. More precisely, for $A \in \Psi \mathrm{DO}_{c l}^{0}(X, E)$, a zero order classical pseudodifferential operator, the principal symbol $\sigma_{A}$ is an element in $C^{\infty}\left(T_{1}^{*} X, \operatorname{End}\left(\pi^{*}(E)\right)\right)$, where $\pi^{*}(E)$ is the pull-back of the bundle $E \rightarrow X$ under the projection $\pi: T_{1}^{*} X \rightarrow X$. Note that the connection $\nabla^{E}$ determines a connection $\nabla$ on $\operatorname{End}\left(\pi^{*}(E)\right)$. Parallel transport along the Hamiltonian flow of $\sigma_{P}$ then determines a flow $\beta_{t}$ acting on $C^{\infty}\left(T_{1}^{*} X, \operatorname{End}\left(\pi^{*}(E)\right)\right)$. Our version of Egorov's theorem specialized to Laplace type operators reads as follows.

Theorem 1.4. If $A \in \Psi \mathrm{DO}_{c l}^{0}(X, E)$ and if $P$ is a positive second order differential operator of Laplace type then for all $t \in \mathbb{R}$ the operators $A_{t}:=e^{+\mathrm{i} t P^{1 / 2}} A e^{-\mathrm{i} t P^{1 / 2}}$ are again in $\Psi_{\mathrm{DO}_{c l}^{0}}^{0}(X, E)$ and $\sigma_{A_{t}}=\beta_{t}\left(\sigma_{A}\right)$.

We actually prove a more general version of this theorem which applies to flows generated by first order pseudodifferential operators with real scalar principal symbols. Note that unlike in the scalar case the first order terms are needed to determine the flow. We show that for pseudodifferential operators with real scalar principal part the subprincipal symbol is invariantly defined as a partial connection along the Hamiltonian vector field, thus allowing us to define all flows without referring to local coordinate systems.

1.1. Discussion. Dirac equation on $\mathbf{R}^{3}$ (and, more generally, on $\mathbf{R}^{d}$ ) has been studied from the semiclassical point of view in the papers BoK98, BoK99, Bol01, BoG04, BoG04.2 of Bolte, Glaser and Keppeler. The authors would like to thank J. Bolte for bringing to their attention this problem on manifolds. Unlike in the works of Bolte, 
Glaser and Keppeler we investigate the high energy limit rather than the semiclassical limit. Therefore, all nontrivial dynamical effects are due to the nontrivial curvature of the spin-connection. This is conceptually different from the stated previous results where quantum ergodicity is due to a spin precession in an external magnetic field. External fields are not seen in the high energy limit and therefore the strict analog of the result of Shnirelman, Colin de Verdière and Zelditch Shn74, Shn93, CV85, Zel87] can not be expected to hold in $\mathbb{R}^{n}$ or on manifolds with integrable geodesic flow.

Apart from working on manifolds our methods also differ from those previously employed as we take the absolute value of the Dirac operator instead of the Dirac operator itself as the generator of the dynamics. This has the advantage of allowing for the full algebra of matrix valued functions as the observable algebra rather than a subalgebra. One can also justify this from a physical point of view. Namely, in a fully quantized theory the generator of the time evolution on the 1-particle Hilbert spaces is the absolute value of the Dirac operator. Furthermore, on the electron 1-particle subspace these two operators coincide.

Our theorem 1.3 for $n=3$ and $k=1$ deals with the electromagnetic field on a 3-dimensional compact manifold. The statement of theorem 1.3 means that quantum ergodicity holds for circular polarized photons if the 2-frame flow is ergodic.

We would also like to mention that the Egorov theorem as we state it is related to a work of Dencker ([D82]), who proved a propagation of singularity theorem for systems of real principal type. It follows from his work that the polarization set of solutions to the Dirac equation is invariant under a certain flow similar to ours. We also refer the reader to [EW96], GMMP97] and references therein, and [San99] for discussion of semiclassical limits for matrix-valued operators, and relations to parallel transport.

Since the high energy limit of the Quantum system associated to Laplace type operators on vector bundles is non-commutative the apropriate language to investigate questions of ergodicity is the language of $C^{*}$-dynamical systems and states (see Appendix (A)). This was already advertized by S. Zelditch in [Zel96] and it is shown there that for a large class of abstract $C^{*}$-dynamical systems classical ergodicity implies quantum ergodicity. The assumptions under which the theorems are stated in Zel96] ( $G$-abelianness or classical abelianness) are in general not satisfied in the examples we study. The method of the proof can be adapted to our situation, however. In our work we identify the classical flows corresponding to the Dirac operator and the Hodge Laplacian as frame flows, which allows us to use the results obtained by Brin, 
Arnold, Pesin, Gromov, Karcher, Burns and Pollicott to exhibit many examples of manifolds where quantum ergodicity holds for Dirac operator, see Corollary 2.1. The connection to their work has not been made before in the literature on quantum ergodicity. Finally, our results on quantum ergodicity for $p$-eigenforms for Hodge Laplacian, and the role played by $2 \min (p, n-p)$-frame flow seem to be completely new. It is a hope of the authors that their results will stimulate further studies of relationship between ergodic theory of partially hyperbolic dynamical systems, and high energy behavior of eigenfunctions of matrix-valued operators.

\section{ERGODIC FRAME FLOWS: KNOWN EXAMPLES}

The $k$-frame flow $\Phi^{t}, k \geq 2$ is defined as follows: let $\left(v_{1}, \ldots, v_{k}\right)$ be an ordered orthonormal set of $k$ unit vectors in $T_{p} X$. Then $\Phi^{t} v_{1}=G^{t} v_{1}$, where $G^{t}$ is the geodesic flow. $\Phi^{t} v_{j}, 2 \leq j \leq k$ translates $v_{j}$ by the parallel translation at distance $t$ along the geodesic determined by $v_{1}$. Here we summarize the cases when the frame flow is known to be ergodic. A $k$-frame flow is a $\mathrm{SO}(k-1)$-extension of the geodesic flow; on an $n$-dimensional manifold, $k$-frame flow is a factor of the $n$-frame flow for $2 \leq k<n$, so ergodicity of the latter implies ergodicity of the former. Frame flow preserves orientation, so in dimension 2 its ergodicity (restricted to positively-oriented frames, say) is equivalent to the ergodicity of the geodesic flow.

Frame flows were considered by Arnold in Arn61. In negative curvature, they were studied by Brin, together with Gromov, Karcher and Pesin, in a series of papers [BrP74, Br75, Br76, BrG80, Br82, BrK84]. Recently, a lot of progress was made in understanding ergodic behavior of general partially hyperbolic systems, including frame flows. In the current paper, the authors are primarily interested in the ergodicity of the flow; the most recent paper dealing with that question appears to be BuP03 by Burns and Pollicott, where the authors establish ergodicity under certain pinched curvature assumptions in "exceptional" dimensions 7 and 8, see below.

In the sequel, we shall assume that $M$ is negatively curved with sectional curvatures satisfying

$$
-K_{2}^{2} \leq K \leq-K_{1}^{2}
$$

The frame flow is known to be ergodic and have the $K$ property

1) if $M$ has constant curvature $\mathrm{Br} 76, \mathrm{BrP} 74$;

2) for an open and dense set of negatively curved metrics (in the $C^{3}$ topology) Br75]; 
3) if $n$ is odd, but not equal to 7 [BrG80]; or if $n=7$ and $K_{1} / K_{2}>$ 0.99023... BuP03;

4) if $n$ is even, but not equal to 8 , and $K_{1} / K_{2}>0.93$, BrK84; or if $n=8$ and $K_{1} / K_{2}>0.99023 \ldots$ BuP03].

By Theorem 4.4 and results of section 5 , we have the following

Corollary 2.1. Quantum ergodicity for Dirac operator and for Hodge Laplacian (conclusions of Theorems 1.1. 1.2 and 1.3) hold in each of the cases (1)-(4).

The frame flow is not ergodic on negatively-curved Kähler manifolds, since the almost complex structure $J$ is preserved. This is the only known example in negative curvature when the geodesic flow is ergodic, but the frame flow is not. In fact, given an orthonormal $k$ frame $\left(v_{1}, \ldots, v_{k}\right)$, the functions $\left(v_{i}, J v_{j}\right), 1 \leq i, j \leq k$ are first integrals of the frame flow, and in some cases it is possible to describe the ergodic components, Br82, BrP74, BrG80]. Note that the conclusion of Theorem 1.2 is false in the Kähler case, because the decomposition into $(p, q)$-forms is a decomposition into invariant subspaces of the LaplaceBeltrami operator. The Kähler case is interesting in its own right and will be discussed in a forthcoming paper.

The frame flow is conjectured to be ergodic whenever the curvature satisfies $-1<K<-1 / 4$, cf. Br82]. That conjecture is still open.

\section{MicRolocAl ANALYSiS FOR OPERATORS ON VECTOR BUNDLES}

3.1. The subprincipal symbol. Let $X$ be a closed manifold. Suppose that $P \in \Psi \mathrm{DO}_{c l}^{m}\left(X, \Lambda^{n / 2} X\right)$. Then the principal symbol $\sigma_{P}$ is well defined as a function on the cotangent bundle $\dot{T}^{*} X=T^{*} X \backslash 0$ which is smooth and positively homogeneous of degree $m$. The subprincipal symbol $\operatorname{sub}(P)$ is defined in local coordinates by

$$
\operatorname{sub}(P):=p_{m-1}-\frac{1}{2 \mathrm{i}} \sum_{j} \frac{\partial^{2} p_{m}}{\partial x^{j} \partial \xi_{j}}
$$

where the functions $p_{m}$ are the terms homogeneous of degree $m$ in the asymptotic expansion of the full symbol of $P$. Surprisingly, the subprincipal symbol turns out to be well defined as a function on $\dot{T}^{*} X$ (see [DH72], ch 5.2). However, the situation changes if we have $P \in \Psi \mathrm{DO}_{c l}^{m}\left(X, \Lambda^{n / 2} X \otimes E\right)$ for some vector bundle $E$. In this case the principal symbol $\sigma_{P}$ is in $C^{\infty}\left(\dot{T}^{*} X\right.$, $\left.\operatorname{End}\left(\pi^{*}(E)\right)\right)$, whereas the subprincipal symbol has a more complicated transformation law under a change of a bundle chart. More explicitly we have with $\phi(x, \xi)=(x, \xi)$ 
for some section $w \in C^{\infty}(X ; E)$ using local coordinates and a local trivialization (see [DH72], Equ. 5.2.2)

$$
\begin{gathered}
e^{-\mathrm{i} \phi} P\left(e^{\mathrm{i} \phi} w\right)=p\left(x, \phi_{x}^{\prime}\right) w-\frac{1}{2 \mathrm{i}} \sum_{j} \frac{\partial^{2} p}{\partial x^{j} \partial \xi_{j}} w \\
+\sum_{j}\left(p_{m}^{(j)}\left(x, \phi_{x}^{\prime}\right) \frac{1}{\mathrm{i}} \frac{\partial w}{\partial x^{j}}+\frac{1}{2 \mathrm{i}} \frac{\partial p_{m}^{(j)}\left(x, \phi_{x}^{\prime}\right)}{\partial x^{j}} w\right) \bmod S^{m-2},
\end{gathered}
$$

where $p_{m}^{(j)}=\frac{\partial p_{m}}{\partial \xi_{j}}$. Suppose now that $p_{m}=\sigma_{P}$ is scalar and real, i.e. $\sigma_{P}(x, \xi)=h(x, \xi) \operatorname{id}_{E_{x}}$ for some $h \in C^{\infty}\left(\dot{T}^{*} X, \mathbb{R}\right)$. The Hamiltonian vector field associated with the principal symbol $\sigma_{P}$ of $P$ is a vector field on $\dot{T}^{*} X$ and defined in local coordinated by

$$
H_{P}=\sum_{j}\left(\frac{\partial \sigma_{P}}{\partial \xi_{j}} \frac{\partial}{\partial x^{j}}-\frac{\partial \sigma_{P}}{\partial x^{j}} \frac{\partial}{\partial \xi_{j}}\right) .
$$

Now $p_{m}^{(j)}\left(x, \phi_{x}^{\prime}\right)$ has a nice interpretation in terms of $H_{P}$. Namely, if $(x, \xi) \in \dot{T}^{*} X$, then $p_{m}^{(j)}(x, \xi)$ is the push forward of $H_{P}(x, \xi)$ under the projection $\pi: \dot{T}^{*} X \rightarrow X$ expressed in local coordinates, i.e.

$$
\sum_{j} p^{(j)}(x, \xi) \frac{\partial}{\partial x^{j}}=\pi_{*}\left(H_{P}(x, \xi)\right) .
$$

Therefore, in case $E$ is trivial the last sum in (2) is exactly the Lie Derivative $-\mathrm{i} \mathcal{L}_{v} w$ of half densities along the vector field $v=\pi_{*}\left(H_{P}\left(x, \phi_{x}^{\prime}\right)\right)$, which is defined without reference to the local coordinate system and depends on the function $\phi$ only. Hence, if we fix a local trivialization of $E$ and change coordinates on the base manifold only, $\sigma_{P}$ and $\operatorname{sub}(\mathrm{P})$ transform as functions in $C^{\infty}\left(\dot{T}^{*} X, \operatorname{End}\left(\pi^{*}(E)\right)\right)$. Note that $\sum_{j} p_{m}^{(j)}\left(x, \phi_{x}^{\prime}\right) \frac{1}{\mathrm{i}} \frac{\partial w}{\partial x^{j}}$ is the only term in (2) which depends on the derivative of $w$. Hence, under a change of bundle charts by the local function $A \in C^{\infty}(X, \mathrm{GL}(E))$ we get the transformation law

$$
\begin{gathered}
\operatorname{sub}(P) \rightarrow A^{-1} \operatorname{sub}(P) A+A^{-1} p^{(j)} \partial_{j} A= \\
=A^{-1} \operatorname{sub}(P) A+A^{-1} \frac{1}{\mathrm{i}} H_{P} A
\end{gathered}
$$

whereas $\sigma_{P}$ transforms as a function in $C^{\infty}\left(\dot{T}^{*} X, \operatorname{End}\left(\pi^{*}(E)\right)\right)$. If the principal symbol is scalar and real (5) is the transformation law of a partial connection ${ }^{2}$ along the vector field $H_{P}$. Hence, by $\nabla_{H_{P}}:=$

\footnotetext{
${ }^{2} \mathrm{~A}$ partial connection along a vector field $v$ can be defined by its covariant derivative which is a map $\nabla_{v}: C^{\infty}(X ; E) \rightarrow C^{\infty}(X ; E)$ satisfying $\nabla_{v}(f g)=v(f) g+f \nabla_{v} g$
} 
$H_{P}+\mathrm{i} \operatorname{sub}(P)$ a covariant derivative is defined. We have therefore proved the following proposition.

Proposition 3.1. Let $E$ be a vector bundle and suppose that $P \in$ $\Psi \mathrm{DO}_{c l}^{m}\left(X, \Lambda^{n / 2} X \otimes E\right)$ has real scalar principal symbol. Then the subprincipal symbol of $\operatorname{sub}(P)$ defined locally by (1) is invariantly defined as a partial connection on $\pi^{*} E$ along the Hamiltonian vector field $H_{P}$.

Let $X$ be an oriented closed $n$-dimensional Riemannian manifold, let $E \rightarrow X$ be a hermitian vector bundle and suppose that $P: C^{\infty}(X ; E) \rightarrow$ $C^{\infty}(X ; E)$ is a formally selfadjoint second order differential operator of Laplace type, i.e. $\sigma_{P}(\xi)=g(\xi, \xi) \cdot \mathbf{1}$. Then there exists a hermitian connection (see e.g. BGV92 ch. 2.1)

$$
\nabla: C^{\infty}(X ; E) \rightarrow C^{\infty}\left(X ; E \otimes T^{*} X\right)
$$

and a potential $V \in C^{\infty}(X ; \operatorname{End}(E))$ such that

$$
P=\nabla^{*} \nabla+V
$$

The connection and the potential are uniquely determined by these properties. The operator $P$ is essentially selfadjoint on $C^{\infty}(X ; E)$ and in case it is positive we may define the square root $P^{1 / 2}$ by functional calculus. By Seeley ([See67]) we know that $P^{1 / 2}$ is a classical pseudodifferential operator of order 1 and its principal symbol is given by $\sigma_{P^{1 / 2}}(\xi)=\|\xi\|_{g} \cdot \mathbf{1}$. We use the metric to identify the bundle $\Lambda^{n / 2} X$ with the trivial bundle and in this way we understand $P$ and $P^{1 / 2}$ as operators acting on $C^{\infty}\left(X ; E \otimes \Lambda^{n / 2} X\right)$. Since $P$ is of Laplace type the Hamiltonian vector field $H_{P^{1 / 2}}$ when restricted to the unit tangent bundle $T_{1}^{*} X$ coincides with the geodesic spray. We will therefore write $H_{g}$ for $H_{P^{1 / 2}}$ in order to emphasize the dependence from the metric.

Now $\nabla$ determines uniquely a hermitian partial connection $\tilde{\nabla}_{H_{g}}$ along $H_{g}$ on $\pi^{*} E$ which satisfies

$$
\left(\tilde{\nabla}_{H_{g}} \pi^{*}(f)\right)(x, \xi)=\nabla_{\pi_{*}\left(H_{g}(x, \xi)\right)} f \quad \text { for all } f \in C^{\infty}(X, E)
$$

where $\pi^{*}(f) \in C^{\infty}\left(T^{*} X ; \pi^{*}(E)\right)$ is the pull back of a section $f \in$ $C^{\infty}(X ; E)$. If we fix a local trivialization of $E$ we have

$$
\left(\tilde{\nabla}_{H_{g}} f\right)(x, \xi)-\left(H_{g} f\right)(x, \xi)=\frac{\mathrm{i}}{\|\xi\|} \sum_{i, k} g^{i k} A_{i} \xi_{k},
$$

where $\nabla_{i}=\partial_{i}+\mathrm{i} A_{i}$. The geometric meaning of this partial connection is as follows. The vector field $H_{g}$ generates the geodesic flow. Hence, a partial connection along $H_{g}$ allows to transport vectors along geodesics.

for all $f \in C^{\infty}(X), g \in C^{\infty}(X ; E)$. Hence, parallel transport is defined along $v$ only. Moreover, where $v$ vanishes this is a bundle homomorphism. 
The partial connection (6) is chosen in such a way that $v \in \pi^{*}(E)_{(x, \xi)}=$ $E_{x}$ gets transported along the geodesic with the original connection $\nabla$ on $E$. We have

Proposition 3.2. For $P=\nabla^{*} \nabla+V$ as above the partial connection determined by the subprincipal symbol $\operatorname{sub}\left(P^{1 / 2}\right)$ of $P^{1 / 2}$ coincides with $\tilde{\nabla}_{H_{g}}$.

Proof. We calculate everything in local coordinates where $|g|=1$ to keep the formulas as simple as possible. In such local coordinates we have $\nabla_{i}=\partial_{i}+\mathrm{i} A_{i}$. Then one easily calculates

$$
\operatorname{sub}(P)(\xi)=\sum_{i, k} 2 \mathrm{i} g^{i k} A_{i} \xi_{k} .
$$

Since the principal symbol of $P$ is scalar the formula as proved in DG75

$$
\operatorname{sub}\left(P^{1 / 2}\right)(\xi)=\frac{1}{2} \sigma_{P}^{-\frac{1}{2}} \operatorname{sub}(P),
$$

continues to hold and we obtain

$$
\operatorname{sub}\left(P^{1 / 2}\right)(\xi)=\mathrm{i}\|\xi\|^{-1} \sum_{i, k} g^{i k} A_{i} \xi_{k}
$$

This coincides with the claimed formula.

3.2. Egorov's theorem. Suppose that $A \in \Psi \mathrm{DO}_{c l}^{1}\left(X ; E \otimes \Lambda^{n / 2}\right)$ has real scalar principal part, let $H_{A}$ be the associated Hamiltonian vector field on $\dot{T}^{*} X$ and let $\nabla_{H_{A}}=H_{A}+\mathrm{i} \operatorname{sub}(A)$ be the partial connection on $\pi^{*} E$ defined by the subprincipal symbol. Then this determines a geometric flow $\alpha_{t}$ on the vector bundle $\pi^{*} E$ such that the flow lines $(x(t), \xi(t), v(t))$ consists of the orbits $(x(t), \xi(t))$ of the Hamiltonian flow and $v(t)$ expressed in coordinates of a local bundle chart satisfies

$$
\frac{d v(t)}{d t}=\mathrm{i} \operatorname{sub}(A) v(t)
$$

Note that $\alpha_{t}$ lifts the Hamiltonian flow $h_{t}$ on $\dot{T}^{*} X$ and makes $\pi^{*} E$ an $\mathbb{R}$-equivariant vector bundle. The induced flow $\alpha_{t}^{*}$ on $C^{\infty}\left(\dot{T}^{*} X, \pi^{*} E\right)$ satisfies

$$
\frac{d}{d t} \alpha_{t}^{*} f=\nabla_{H_{A}} f
$$

which shows that the flow is defined independent of a choice of local coordinates. Hence, there is also an action $\operatorname{Ad}\left(\alpha_{t}\right)$ of $\mathbb{R}$ on $\pi^{*} \operatorname{End}(E)$ which extends the Hamiltonian flow and is compatible with $\alpha_{t}$. This 
defines a flow on $C^{\infty}\left(\dot{T}^{*} X, \pi^{*} \operatorname{End}(E)\right)$ which we denote by $\operatorname{Ad}\left(\alpha_{t}\right)^{*}$. Clearly, if $f \in C^{\infty}\left(\dot{T}^{*} X, \pi^{*} \operatorname{End}(E)\right)$

$$
\frac{d}{d t} \operatorname{Ad}\left(\alpha_{t}\right)^{*} f=\left[\nabla_{H_{A}}, f\right]
$$

In local coordinates one has

$$
\left[\nabla_{H_{A}}, f\right]=H_{A} f+\mathrm{i}[\operatorname{sub}(A), f] .
$$

Egorov's theorem now reads as follows.

Proposition 3.3. Let $A \in \Psi \mathrm{DO}_{c l}^{1}\left(X ; E \otimes \Lambda^{n / 2}\right)$ and suppose that the principal symbol of $A$ is of real scalar type, i.e. $\sigma_{A}(\xi)=h(\xi) \cdot \mathbf{1}$, where $h \in C^{\infty}\left(\dot{T}^{*} M, \mathbb{R}\right)$. Then, if $B$ is in $\Psi_{D^{\prime}}^{m}\left(X ; E \otimes \Lambda^{n / 2}\right)$, also $B_{t}:=e^{+\mathrm{i} t A} B e^{-\mathrm{i} t A}$ is in $\Psi \mathrm{DO}_{c l}^{m}(X ; E)$. Moreover,

$$
\sigma_{B_{t}}=\operatorname{Ad}\left(\alpha_{t}\right)^{*}\left(\sigma_{B}\right) .
$$

Proof. As usual we have

$$
\frac{d}{d t} B_{t}=\mathrm{i}[A, B]
$$

The right hand side is a pseudodifferential operator in $\Psi \mathrm{DO}_{c l}^{n}(X ; E \otimes$ $\Lambda^{n / 2}$ ) and its principal symbol is given by

$$
\sigma_{\mathrm{i}[A, B]}=\left\{\sigma_{A}, \sigma_{B}\right\}+\mathrm{i}[\operatorname{sub}(A), B] .
$$

This follows immediately from the formulas for the asymptotic expansion of products of pseudodifferential operators. By (11) and (12) equation (13) is on the level of principal symbols the flow equation for $\operatorname{Ad}\left(\alpha_{t}\right)^{*}$. This equation can be solved on the symbol level order by order and one can construct a classical symbol for $B_{t}$ in the usual way (as for example carried out in Tay81, Ch VIII, §1). The only difference to the scalar case is the second term in (14) which causes the Hamiltonian flow to be replaced by $\operatorname{Ad}\left(\alpha_{t}\right)^{*}$.

Suppose now that $X$ is an oriented Riemannian manifold and let $A=P^{1 / 2}$, where $P=\nabla^{*} \nabla+V$ is a positive Laplace type operator. We may use the metric to identify $\Lambda^{1 / 2} X$ with the trivial bundle. Moreover, the Hamiltonian vector field $H_{A}$ restricted to the unit cotangent bundle $T_{1}^{*} X$ coincides with the geodesic spray. In this case it is convenient to identify positively homogeneous functions on $\dot{T}^{*} X$ with smooth functions on $T_{1}^{*} X$ by restriction. Hence, the principal symbol of an operator in $\Psi \mathrm{DO}_{c l}^{m}\left(X ; E \otimes \Lambda^{n / 2}\right)$ is in $C^{\infty}\left(T_{1}^{*} X, \pi^{*} \operatorname{End}(E)\right)$. In this case the Egorov theorem says, that the flow $\alpha_{t}$ transports vectors in $\pi^{*} E$ parallel with respect to the connection $\nabla$ along the geodesic flow on $T_{1}^{*} X$. 


\section{The Dirac operator And the frame Flow}

In this section $(X, g)$ is a compact oriented Riemannian manifold of dimension $n \geq 3$. A spin structure (see e.g. [LM89, Fri]) on $X$ is an $\operatorname{Spin}(n)$-principal bundle $P$ over $X$ together with a smooth covering $\eta$ from $P$ onto the bundle $F X$ of oriented orthonormal frames, such that the following diagram is commutative.

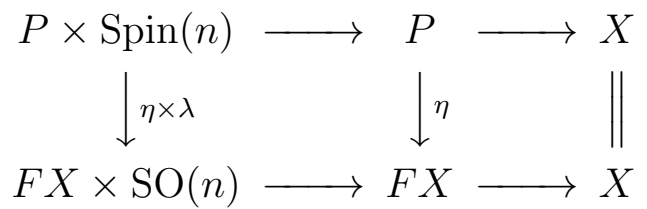

Here $\lambda$ denotes the covering map $\operatorname{Spin}(n) \rightarrow \mathrm{SO}(n)$. The (complexified) Clifford algebra $\mathrm{Cl}_{c}\left(\mathbb{R}^{n}\right)$ is isomorphic to $\operatorname{Mat}\left(2^{\left[\frac{n}{2}\right]}, \mathbb{C}\right)$ if $n$ is even and to $\operatorname{Mat}\left(2^{\left[\frac{n}{2}\right]}, \mathbb{C}\right) \oplus \operatorname{Mat}\left(2^{\left[\frac{n}{2}\right]}, \mathbb{C}\right)$ if $n$ is odd. The Clifford modules $\Delta_{n}$ are then defined by the action of this matrix algebra on $\mathbb{C}^{2^{\left[\frac{n}{2}\right]}}$, where in case $n$ is odd we project onto the first summand. Hence, $\Delta_{n}$ is an irreducible module for the Clifford algebra $\mathrm{Cl}_{c}\left(\mathbb{R}^{n}\right)$. Since $\operatorname{Spin}(n) \subset \mathrm{Cl}_{c}\left(\mathbb{R}^{n}\right)$ the Clifford modules $\Delta_{n}$ are also modules for the group $\operatorname{Spin}(n)$. The corresponding representation $\rho: \operatorname{Spin}(n) \rightarrow \operatorname{Aut}\left(\Delta_{n}\right)$ is called the spinor representation of $\operatorname{Spin}(n)$. This representation is irreducible if $n$ is odd. It is the direct sum of two irreducible components if $n$ is even. The spinor bundle $S$ associated with a Spin structure is the associated bundle $P \times{ }_{\rho} \Delta_{n}$. The Levi-Civita connection on $F X$ lifts naturally to a connection on $P$ and this defines a connection $\nabla_{S}: C^{\infty}(X ; S) \rightarrow C^{\infty}\left(X ; S \otimes T^{*} X\right)$ on $S$, the Levi-Civita connection on the spinor bundle. The Dirac operator $D: C^{\infty}(X ; S) \rightarrow C^{\infty}(X ; S)$ is defined by $-\mathrm{i} \gamma \circ \nabla_{S}$, where $\gamma$ denotes the action of covector fields on sections of the spinor bundle by Clifford multiplication. $D$ is of Dirac type and essentially selfadjoint on $C^{\infty}(X ; S)$. The operator $F=\operatorname{sign}(D)$ is in $\Psi \mathrm{DO}_{c l}^{0}(X ; S)$ and its principal symbol $\sigma_{F}(\xi)$ is given by Clifford multiplication by $\frac{1}{|\xi|} \xi$. If $n$ is even, then Clifford multiplication with the volume form times $\mathrm{i} \frac{n(n+1)}{2}$ defines an involution $\Gamma$ on $L^{2}(X ; S)$ which anti-commutes with $D$ and with $F$, but which commutes with $|D|$. The Lichnerowicz formula allows us to express the square of the Dirac operator by the spinor Laplacian

$$
D^{2}=\nabla_{S}^{*} \nabla_{S}+\frac{1}{4} R
$$

where $R$ is the scalar curvature. By Prop. 3.2 the connection determined by the subprincipal symbol $\operatorname{sub}(|D|)$ of $|D|$ transports spinors along geodesics with the spinor connection $\nabla_{S}$. The corresponding flow 
on the bundle $\pi^{*} S \rightarrow T_{1}^{*} X$ will be denoted by $\alpha_{t}$. The induced flow on the bundle $\pi^{*} \operatorname{End}(S) \rightarrow T_{1}^{*} X$ will be denoted as before by $\operatorname{Ad}\left(\alpha_{t}\right)$. This induces a 1-parameter group $\beta_{t}=\operatorname{Ad}\left(\alpha_{t}\right)^{*}$ of $*$-automorphisms of the algebra $C^{\infty}\left(T_{1}^{*} X, \pi^{*} \operatorname{End}(S)\right)$. It extends continuously to the $C^{*}$ algebra $\mathcal{A}=C\left(T_{1}^{*} X, \pi^{*} \operatorname{End}(S)\right)$. The Egorov theorem of the previous section therefore reads as follows.

Proposition 4.1. Let $D$ be the Dirac operator on a compact spin manifold $X$ and let $A \in \Psi \mathrm{DO}_{c l}^{0}(X ; S)$. Then with $A_{t}:=e^{+\mathrm{i} t|D|} A e^{-\mathrm{i} t|D|}$ we have $A_{t} \in \Psi \operatorname{DO}_{c l}^{0}(X ; S)$ for all $t \in \mathbb{R}$ and

$$
\sigma_{A_{t}}=\beta_{t}\left(\sigma_{A}\right)
$$

And as a consequence we get

Corollary 4.2. Let $X$ be a closed Riemannian spin manifold of dimension $n \geq 3$ and let $D$ be the Dirac operator. Let $\phi_{k}$ be a sequence of eigensections to $D^{2}$ with

$$
D^{2} \phi_{k}=\lambda_{k} \phi_{k}, \quad\left\langle\phi_{k}, \phi_{j}\right\rangle=\delta_{k j} .
$$

such that $\left|\lambda_{k}\right| \nearrow \infty$ and such that the sequence of states

$$
\omega_{i}(A):=\left\langle\phi_{i}, A \phi_{i}\right\rangle
$$

on the $C^{*}$-algebra $\overline{\Psi \mathrm{DO}_{c l}^{0}(X ; S)}$ converges in the weak-*-topology. Then there is a $\beta_{t}$-invariant state $\omega_{\infty}$ on $C\left(T_{1}^{*} X, \pi^{*} \operatorname{End}(S)\right)$ such that

$$
\lim _{n \rightarrow \infty} \omega_{n}(A)=\omega_{\infty}\left(\sigma_{A}\right)
$$

Proof. Since the states $\omega_{i}$ are invariant under the flow induced by conjugation with $e^{\mathrm{i}|D| t}$ so is the limit state. The limit state $\omega$ vanishes on all operators of order -1 since their product with $|D|$ is bounded. Since the norm closure of those operators are the compact operators $\mathcal{K}$ we have $\omega(\mathcal{K})=\{0\}$. Hence, the state projects to a state on the quotient

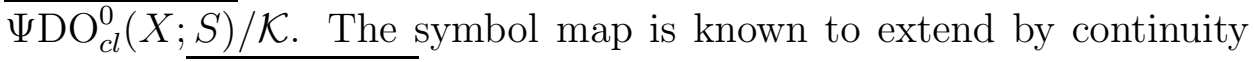
to a map $\overline{\Psi \mathrm{DO}_{c l}^{0}(X ; S)} \rightarrow C\left(T_{1}^{*} X, \pi^{*} \operatorname{End}(S)\right)$ with kernel $\mathcal{K}$. Hence, the quotient is via the symbol map isomorphic to $C\left(T_{1}^{*} X, \pi^{*} \operatorname{End}(S)\right)$. Moreover, by the above the symbol map is equivariant with respect to the two flows.

There are two natural invariant states $\omega_{ \pm}$on $C\left(T_{1}^{*} X, \pi^{*} \operatorname{End}(S)\right)$ given by

$$
\omega_{ \pm}(a):=\frac{2}{\operatorname{rk}(S)} \int_{T_{1}^{*} X} \operatorname{Tr}\left(P_{ \pm}(\xi) a(\xi) P_{ \pm}(\xi)\right) d \mu_{L}(\xi)
$$


where $\mu_{L}$ is the normalized Liouville measure and $P_{ \pm}:=\frac{1}{2}\left(1 \pm \sigma_{F}\right)$. The tracial state

$$
\begin{gathered}
\omega=\frac{1}{2}\left(\omega_{+}+\omega_{-}\right) \\
\omega(a)=\frac{1}{\operatorname{rk}(S)} \int_{T_{1}^{*} X} \operatorname{Tr}(a(\xi)) d \mu_{L}(\xi)
\end{gathered}
$$

is therefore not ergodic (see Appendix $\mathrm{A}$ for the notion of ergodicity in this context).

Theorem 4.3. Suppose the frame flow is ergodic. Then $\omega_{+}$and $\omega_{-}$ are ergodic states with respect to $\beta_{t}$. If moreover $n$ is odd the systems $\left(C\left(T_{1}^{*} X, \pi^{*} \operatorname{End}(S)\right), \omega_{ \pm}\right)$are $\beta_{t}$-abelian.

Proof. Note that since the Levi-Civita connection is compatible with the Clifford multiplication both $P_{+}$and $P_{-}$are easily seen to be invariant under $\beta_{t}$. To prove ergodicity we have to show (see Appendix A) that all $\beta_{t}$-invariant elements in $P_{ \pm} L^{\infty}\left(T_{1}^{*} X, \pi^{*} \operatorname{End}(S)\right) P_{ \pm}$are of the form $c P_{ \pm}$with $c \in \mathbb{C}$. To show $\beta_{t}$-abelianness we have to show that an invariant element in $L^{2}\left(T_{1}^{*} X, \pi^{*} \operatorname{End}(S)\right)$ is of the form $c_{1} P_{+}+c_{2} P_{-}$ with $c_{1}, c_{2} \in \mathbb{C}$. We therefore analyze, how invariant elements in $L^{2}\left(T_{1}^{*} X, \pi^{*} \operatorname{End}(S)\right)$ look like.

Step 1: Denote by $\mathbb{C}\left\langle X_{1}, \ldots, X_{n}\right\rangle$ the space of noncommutative polynomials in the variables $X_{1}, \ldots, X_{n}$. Now we define a continuous map

$$
\begin{gathered}
T: L^{2}(F X) \otimes \mathbb{C}\left\langle X_{1}, \ldots, X_{n}\right\rangle \rightarrow L^{2}\left(T_{1}^{*} X, \pi^{*} \operatorname{End}(S)\right), \\
T(f \otimes p)(\xi):=\int_{F_{\xi} X} f(\xi, v) p(\xi, v) d \mu(v),
\end{gathered}
$$

where the integration is over the invariant measure on the fiber $F_{\xi} X$. The action of covectors on spinors here is by Clifford multiplication. The pullback $h_{t}^{*}$ of the frame flow on $F X$ defines a flow on $L^{2}(F X) \otimes$ $\mathbb{C}\left\langle X_{1}, \ldots, X_{n}\right\rangle$ by acting on the first tensor factor. Since the connection is compatible with Clifford multiplication and the Clifford action the map $T$ intertwines the pullback $h_{t}^{*}$ of the frame flow and the flow $\beta_{t}$, i.e.

$$
T \circ\left(h_{t}^{*} \otimes \mathbf{1}\right)=\beta_{t} \circ T .
$$

Note that the Clifford action on the spinor bundle is irreducible. This implies that $T$ is onto.

Step 2: Let $\Psi \in L^{2}\left(T_{1}^{*} X, \pi^{*} \operatorname{End}(S)\right)$ be an invariant vector. By the above we can choose an element $f \in L^{2}(F X) \otimes \mathbb{C}\left\langle X_{1}, \ldots, X_{n}\right\rangle$ such that $T(f)=\Psi$. Since by assumption the frame flow is ergodic we have $\lim _{T \rightarrow \infty} \frac{1}{T} \int_{0}^{T} h_{t}^{*}(f) d t=\int_{F X} f(x) d x=m$, where $m$ is a constant 
polynomial. Since $\Psi$ is invariant and $T$ is equivariant we have almost everywhere

$$
\Psi(\xi)=T(m)(\xi)=\left(\int_{F_{\xi} X} m(\xi, v) d \mu(v)\right) .
$$

Since the measure on $F_{\xi} X$ is invariant under the action of $S O(n-$ $1)$ the endomorphism $\int_{F_{\xi} X} m(\xi, v) d \mu(v)$ commutes with the action of $\operatorname{Spin}(n-1)$. Clearly, the projections $P_{+}(\xi)$ and $P_{-}(\xi)$ commute with the $\operatorname{Spin}(n-1)$ action, and it is easy to see (for example by calculating the dimensions) that the representations of $\operatorname{Spin}(n-1)$ on the ranges of $P_{+}(\xi)$ and $P_{-}(\xi)$ are irreducible. Moreover, these two representations are equivalent iff $n$ is even. In this case the algebra of invariant matrices in $\operatorname{End}\left(\mathrm{S}_{\xi}\right)$ is generated by $P_{+}(\xi), P_{-}(\xi), \Gamma$. This shows that an invariant element of the form $P_{ \pm} A P_{ \pm}$is proportional to $P_{ \pm}$which proves ergodicity. If $n$ is odd the two irreducible representations of $\operatorname{Spin}(n-1)$ on the ranges of $P_{+}(\xi)$ and $P_{-}(\xi)$ are inequivalent. Hence, any invariant element in $A \in \operatorname{End}\left(\mathrm{S}_{\xi}\right)$ is of the form $c_{1} P_{+}+c_{2} P_{-}$.

Remark: That $\left(C\left(T_{1}^{*} X, \pi^{*} \operatorname{End}(S)\right), \omega_{ \pm}\right)$is not $\beta_{t}$-abelian in even dimensions has a simple interpretation. It is that the space of invariant states on $C\left(T_{1}^{*} X, \pi^{*} \operatorname{End}(S)\right)$ is not a simplex, i.e. the decomposition of an invariant state into ergodic states is not unique. For example the tracial state $\omega$ has the decomposition $\omega=\frac{1}{2}\left(\omega_{+}+\omega_{-}\right)$, but it also has the decomposition $\omega=\frac{1}{2}\left(\omega_{1}+\omega_{2}\right)$ where $\omega_{1}(a)=\omega((1+\Gamma) a)$ and $\omega_{2}(a)=\omega((1-\Gamma) a)$. The states $\omega_{1}$ and $\omega_{2}$ can also be shown to be ergodic if the frame flow is ergodic.

Theorem 4.4. Let $X$ be a compact Riemannian spin manifold with spinor bundle $S$ and Dirac operator $D: C^{\infty}(X ; S) \rightarrow C^{\infty}(X ; S)$. Let $\phi_{k}$ be an orthonormal sequence of eigensections of $D$ with eigenvalues $\lambda_{k} \nearrow \infty$ such that $\phi_{k}$ spans $^{3} L_{+}^{2}(X ; S)=\frac{1+F}{2} L^{2}(X ; S)$. If the frame flow of $X$ is ergodic, then Quantum Ergodicity holds, i.e.

$$
\lim _{N \rightarrow \infty} \frac{1}{N} \sum_{k=1}^{N}\left|\left\langle\phi_{k}, A \phi_{k}\right\rangle-\omega_{+}\left(\sigma_{A}\right)\right|=0, \quad \text { for all } A \in \Psi \operatorname{DO}_{c l}^{0}(X ; S) .
$$

Moreover, there is a density one subsequence $\phi_{k}^{\prime}$ such that

$$
\lim _{k \rightarrow \infty}\left\langle\phi_{k}^{\prime}, A \phi_{k}^{\prime}\right\rangle=\omega_{+}(\sigma(A)), \quad \text { for all } A \in \Psi \operatorname{DO}_{c l}^{0}(X ; S) .
$$

An analogous statement holds for eigensections with $\lambda_{k} \searrow-\infty$ with $\omega_{+}$replaced by $\omega_{-}$.

\footnotetext{
${ }^{3}$ in the sense that the hull of the vectors is dense.
} 
Proof. We denote $\omega=\frac{1}{2}\left(\omega_{+}+\omega_{-}\right)=\frac{1}{\operatorname{rk}(S)} \int_{T_{1}^{*} X} \operatorname{Tr}\left(\sigma_{A}(\xi)\right) d \mu(\xi)$ be the ordinary tracial state on $C^{\infty}\left(T_{1}^{*} X, \pi^{*} \operatorname{End}(S)\right)$. The heat trace asymptotics (cf. GrSee95)

$$
\operatorname{Tr}\left(A \cdot e^{-D^{2} t}\right)=C(n) \int_{T_{1}^{*} X} \operatorname{Tr}\left(\sigma_{A}(\xi)\right) d \mu(\xi) \cdot t^{-n / 2}+O\left(t^{-n / 2+1 / 2}\right)
$$

which one easily gets from the classical calculus of pseudodifferential operators together with Karamata's Tauberian theorem implies that

$$
\lim _{N \rightarrow \infty} \frac{1}{N} \sum_{k=1}^{N}\left\langle\frac{1+F}{2} \phi_{k}, A \frac{1+F}{2} \phi_{k}\right\rangle=\omega_{+}\left(\sigma_{A}\right), \quad A \in \Psi \operatorname{DO}_{c l}^{0}(X ; S) .
$$

The proof is now an analog to the proof of Shnirelman, Zelditch and Colin de Verdière ( Shn74, Shn93, CV85, Zel87]). To prove Quantum ergodicity it is obviously enough to show that for any selfadjoint $A \in$ $\Psi \mathrm{DO}_{c l}^{0}(X ; S)$ with $\omega_{+}\left(\sigma_{A}\right)=0$ we have

$$
\lim _{N \rightarrow \infty} \frac{1}{N} \sum_{k=1}^{N}\left|\left\langle\phi_{k}, A \phi_{k}\right\rangle\right|=0 \text {. }
$$

Clearly, $\left|\left\langle\phi_{k}, A \phi_{k}\right\rangle\right|=\left|\left\langle\phi_{k}, \frac{1+F}{2} A \frac{1+F}{2} \phi_{k}\right\rangle\right|$ and therefore, we assume without loss of generality that $A=\frac{1+F}{2} A \frac{1+F}{2}$. Hence, $\sigma_{A}=P_{+} \sigma_{A} P_{+}$. Now let $A_{T}:=\frac{1}{T} \int_{0}^{T} e^{+\mathrm{i} t|D|} A e^{-\mathrm{i} t|D|} d t$ and since $\omega_{+}$is ergodic we have $\omega_{+}\left(\left|\sigma_{A_{T}}\right|^{2}\right) \rightarrow 0$ (see Lemma A.1) as $T \rightarrow \infty$. This implies that also $\omega_{+}\left(\left|\sigma_{A_{T}}\right|\right) \rightarrow 0$ as $T \rightarrow \infty$. On the other hand we have

$$
\left|\left\langle\phi_{k}, A \phi_{k}\right\rangle\right|=\left|\left\langle\phi_{k}, A_{T} \phi_{k}\right\rangle\right| \leq\left\langle\phi_{k},\left|A_{T}\right| \phi_{k}\right\rangle \text {. }
$$

Then formula (22) applied to $\left|A_{T}\right|$ together with the fact that $\omega_{+}\left(\sigma_{\left|A_{T}\right|}\right)<$ $\epsilon$ for $T$ large enough allows us to conclude that

$$
\limsup _{N \rightarrow \infty} \frac{1}{N} \sum_{k=1}^{N}\left|\left\langle\phi_{k}, A \phi_{k}\right\rangle\right|<\epsilon,
$$

for all $\epsilon>0$ from which the assertion follows. The existence of a density one sequence is based on a diagonalization argument which is well known (see e.g. [Zel96]).

\section{The Hodge-Laplace}

Let $X$ be a compact oriented Riemannian manifold, let $\Lambda^{*} X:=$ $\Lambda^{*} T^{*} X$ be the exterior algebra bundle, and let $\Lambda_{\mathbb{C}}^{*} X$ be its complexification. We denote by $d$ the exterior derivative, $\delta$ the coderivative (i.e. the formal adjoint of $d$ ), and by $*$ the Hodge star operator. Then $d+\delta$ 
is a Dirac type operator acting on sections of $\Lambda_{\mathbb{C}}^{*} X$. Its square is the Hodge Laplace operator

$$
\Delta=d \delta+\delta d
$$

Note that $\Delta$ leaves the subspace of $p$-forms invariant. In the following we will denote the restriction of $\Delta$ to $p$-forms by $\Delta_{p}$. The ordinary Laplace operator acting on functions is therefore equal to $\Delta_{0}$. If $\nabla_{p}$ is the Levi-Civita covariant derivative of $p$-forms the Weitzenböck formula states that

$$
\Delta_{p}=\nabla_{p}^{*} \nabla_{p}+H_{p}
$$

where $H_{p}$ is section of $\operatorname{End}\left(\Lambda^{p} T^{*} X\right)$ which can be expressed in terms of the curvature of the connection. For example $H_{1}$ is equal to the Ricci curvature. We conclude that the partial connection determined by the subprincipal symbol of $\Delta_{p}^{1 / 2}$ transports $p$-multivectors along the Hamiltonian vector field parallel with respect to the Levi-Civita connection. The corresponding flow on the $C^{*}$-algebra $C\left(T_{1}^{*} X, \pi^{*} \operatorname{End}\left(\Lambda_{\mathbb{C}}^{p} X\right)\right)$ will be denoted by $\beta_{t}$. There is a natural invariant tracial state $\omega_{\text {tr }}$ on $C\left(T_{1}^{*} X, \pi^{*} \operatorname{End}\left(\Lambda_{\mathbb{C}}^{p} X\right)\right)$ given by

$$
a \rightarrow \omega_{\operatorname{tr}}(a)=\left(\begin{array}{c}
n \\
p
\end{array}\right)^{-1} \int_{T_{1}^{*} X} \operatorname{Tr}(a(\xi)) d L(\xi) .
$$

As in the Dirac case this state is not ergodic for $0<p<n$. Let $P \in C\left(T_{1}^{*} X, \pi^{*} \operatorname{End}\left(\Lambda_{\mathbb{C}}^{p} X\right)\right)$ defined by

$$
P(\xi) v:=i(\xi) \xi \wedge v
$$

where $i(\xi)$ denotes the operator of interior multiplication with $\xi$. Then $P$ is an orthogonal projection in $C\left(T_{1}^{*} X, \pi^{*} \operatorname{End}\left(\Lambda_{\mathbb{C}}^{p} X\right)\right)$ which is invariant under $\beta_{t}$, and hence

$$
\begin{aligned}
\omega_{\mathrm{tr}} & =\frac{n-p}{n} \omega_{t}+\frac{p}{n} \omega_{l}, \\
\omega_{l}(a) & =\frac{n}{p} \omega_{\mathrm{tr}}((1-P) \cdot a), \\
\omega_{t}(a) & =\frac{n}{n-p} \omega_{\mathrm{tr}}(P \cdot a),
\end{aligned}
$$

is a decomposition into invariant states. The non-ergodicity of this state can be seen as the classical counterpart of the Hodge decomposition

$$
C^{\infty}\left(X ; \Lambda_{\mathbb{C}}^{p} X\right)=d C^{\infty}\left(X ; \Lambda_{\mathbb{C}}^{p-1} X\right) \oplus \delta C^{\infty}\left(X ; \Lambda_{\mathbb{C}}^{p+1} X\right) \oplus \operatorname{ker}\left(\Delta_{p}\right)
$$

which induces a decomposition of $L^{2}\left(X ; \Lambda_{\mathbb{C}}^{p} X\right)$ into invariant subspaces of $\Delta_{p}$. 
Theorem 5.1. Suppose $0<p<n, n \geq 3$ and let $k_{p}=2 \min (p, n-p)$. Suppose that the $k_{p}$-frame flow is ergodic and that $p \neq \frac{n-1}{2}$. Then $\omega_{t}$ is an ergodic state with respect to $\beta_{t}$ on $C\left(T_{1}^{*} X, \pi^{*} \Lambda_{\mathbb{C}}^{p} X\right)$. If moreover $p \neq \frac{n}{2}$ then the system $\left(C\left(T_{1}^{*} X, \pi^{*} \Lambda_{\mathbb{C}}^{p} X\right), \omega_{t}\right)$ is $\beta_{t}$-abelian.

Proof. The proof is similar to the proof in the Dirac case. We investigate the set of invariant vectors in $P L^{2}\left(T_{1}^{*} X, \pi^{*} \operatorname{End}\left(\Lambda_{\mathbb{C}}^{p} X\right)\right) P$ and in $L^{2}\left(T_{1}^{*} X, \pi^{*} \operatorname{End}\left(\Lambda_{\mathbb{C}}^{p} X\right)\right) P$.

Step 1: Let $N=k_{p}$. Denote by $\mathcal{P} \subset \mathbb{C}\left\langle X_{1}, \ldots, X_{N}, Y_{1}, \ldots, Y_{N}\right\rangle$ the ring of polynomials in the noncommutative variables $X_{1}, \ldots, X_{N}$ and $Y_{1}, \ldots, Y_{N}$ such that in each summand the same number of $X$ and $Y$ occur. Now define a map

$$
\begin{gathered}
T: L^{2}\left(F_{N} X\right) \otimes \mathcal{P} \rightarrow L^{2}\left(T_{1}^{*} X, \pi^{*} \operatorname{End}\left(\Lambda_{\mathbb{C}}^{p} X\right)\right), \\
T(f \otimes p)(\xi)=\int_{F_{N, \xi} X} f(\xi, v) \hat{p}(v) d \mu(v),
\end{gathered}
$$

where integration is over the fibre $F_{N, \xi} X$ of the bundle $F_{N} X$ over the point $\xi \in T_{1}^{*} X$. The endomorphism $\hat{p}(v)$ is defined by replacing all $X_{i}$ by exterior multiplication with $v_{i}$ and all $Y_{i}$ by interior multiplication with $v_{i}$. Since the number of $X$ and $Y$ is the same in each summand the operators leave the space $\Lambda_{\mathbb{C}}^{p} T_{\pi(\xi)}^{*} X$ invariant. Since exterior and interior multiplication are compatible with the Levi-Civita connection the map $T$ intertwines the pullback of $N$-frame flow and the flow $\beta_{t}$, i.e.

$$
T \circ\left(h_{t}^{*} \otimes \mathbf{1}\right)=\beta_{t} \circ T .
$$

Moreover, by an elementary exercise in linear algebra any endomorphism of $\Lambda_{\mathbb{C}}^{p} T_{\pi(\xi)}^{*} X$ can be represented by a linear combination of elements of the form $\hat{p}\left(v_{1}, \ldots, v_{N}\right)$, where $v_{1}, \ldots, v_{N}$ is a frame and $p$ has degree at most $2 N$. Therefore, the map $T$ is surjective.

Step 2: Now let $\Psi$ be an invariant element in $L^{2}\left(T_{1}^{*} X, \pi^{*} \operatorname{End}\left(\Lambda_{\mathbb{C}}^{p} X\right)\right)$. Then we may find an $f \in L^{2}\left(F_{N} X\right) \otimes \mathcal{P}$ such that $\Psi=T(f)$. By the same argument as in the proof of theorem 4.3 it follows that $\Psi=T(\mathrm{~m})$, where $m$ is some constant polynomial such that $\hat{m}(\xi)$ commutes with the action of $S O(n-1)$ on each fiber of $\pi^{*}\left(\Lambda_{\mathbb{C}}^{p} X\right)$. Note that $P(\xi)$ and $(1-P(\xi))$ project onto invariant subspaces. The fiber of $\pi^{*}\left(\Lambda_{\mathbb{C}}^{p} X\right)$ at the point $(x, \xi) \in T_{1}^{*} X$ is given by $\Lambda_{\mathbb{C}}^{p} T_{x}^{*} X$. On the other hand $T_{x}^{*} X=\mathbb{R} \xi \oplus V$, where $V$ is the orthogonal complement of $\xi$ in $T_{x}^{*} X$. Hence, we have the decomposition $\Lambda^{p} T_{x}^{*} X=\mathbb{R} \otimes \Lambda^{p-1} V \oplus \Lambda^{p} V$. It is now easy to see that $1-P$ projects onto $\mathbb{C} \otimes \Lambda_{\mathbb{C}}^{p-1} V$, whereas $P$ projects onto $\Lambda_{\mathbb{C}}^{p} V$. The representation of $S O(n-1)$ on $\Lambda^{p} \mathbb{C}^{n-1}$ is irreducible (see e.g. [FuHa91], Lecture 18) since we assumed $p \neq \frac{n-1}{2}$. If $p \neq \frac{n}{2}$ 
then all other components which occur in the decomposition into irreducible representations are inequivalent to this representation as one can see by calculating the dimensions (the exceptional case $n=3, p=2$ by other methods). Hence, in this case the algebra of invariant elements in $\operatorname{End}\left(\Lambda_{\mathbb{C}}^{p} T_{x}^{*} X\right)$ is generated by $P(\xi)$ and $1-P(\xi)$. Therefore, any invariant element is of the form $c_{1} P+c_{2}(1-P)$. This shows $\mathbb{R}$-abelianness and ergodicity if $p \neq \frac{n}{2}$. Ergodicity for $p=\frac{n}{2}$ follows from the fact that any invariant element in $P(\xi) \operatorname{End}\left(\Lambda_{\mathbb{C}}^{p} T_{x}^{*} X\right) \stackrel{P}{P}(\xi)$ is proportional to $P(\xi)$ which is a simple consequence of the irreducibility of the $S O(n-1)$ action on $\Lambda_{\mathbb{C}}^{p} V$.

The orthoprojection onto the closure of $\delta C^{\infty}\left(X ; \Lambda^{p-1} T^{*} X\right)$ is given by the zero order pseudodifferential operator $\left(\left.\Delta_{p}\right|_{\operatorname{ker}\left(\Delta_{p}\right)^{\perp}}\right)^{-1} \delta d$. The principal symbol of this operator is easily seen to coincide with $P$. Because of the Hodge decomposition quantum ergodicity for the operator $\Delta_{p}$ with $0<p<n$ can never hold in the strict sense. An eigenform $\phi$ of $\Delta_{p}$ with nonzero eigenvalue can be decomposed uniquely as

$$
\phi=d \phi_{-}+\delta \phi_{+},
$$

where $\phi_{-}$is an eigenform of $\Delta_{p-1}$ and $\phi_{+}$is an eigenform of $\Delta_{p+1}$. Hence, part of the spectrum of $\Delta_{p}$ comes from part of the spectrum of $\Delta_{p-1}$. The other part can be obtained by solving the system

$$
\begin{aligned}
\Delta_{p} \phi & =\lambda \phi, \\
\delta \phi & =0 .
\end{aligned}
$$

We will show that in certain situations this system is quantum ergodic.

Theorem 5.2. Assume $0<p<n, n \geq 3$ and suppose that $\phi_{k}$ is an orthonormal sequence of eigen-p-forms satisfying

$$
\begin{gathered}
\Delta_{p} \phi_{k}=\lambda_{k} \phi_{k}, \\
\delta \phi_{k}=0,
\end{gathered}
$$

such that the $\phi_{k}$ span $\operatorname{ker}(\delta)$ and $\lambda_{k} \nearrow \infty$. Suppose that $p \neq \frac{n-1}{2}$. Then, if the $2 \min (p, n-p)$-frame flow is ergodic, the system is quantum ergodic in the sense that

$$
\lim _{N \rightarrow \infty} \frac{1}{N} \sum_{k \leq N}\left|\left\langle\phi_{k}, A \phi_{k}\right\rangle-\omega_{t}\left(\sigma_{A}\right)\right|=0,
$$

for all $A \in \Psi \mathrm{DO}_{c l}^{0}\left(X ; \Lambda_{\mathbb{C}}^{p} X\right)$. In particular there is a density one subsequence $\phi_{k}^{\prime}$ such that

$$
\lim _{k \rightarrow \infty}\left\langle\phi_{k}^{\prime}, A \phi_{k}^{\prime}\right\rangle=\omega_{t}\left(\sigma_{A}\right), \quad \text { for all } A \in \Psi \mathrm{DO}_{c l}^{0}\left(X ; \Lambda_{\mathbb{C}}^{p} X\right) .
$$


Proof. The proof is along the same lines as the proof of theorem 4.4 Suppose that $A \in \Psi \mathrm{DO}_{c l}^{0}\left(X ; \Lambda_{\mathbb{C}}^{p} X\right)$ with $\omega_{t}(A)=0$ and $A^{*}=A$. Let $F$ be the operator $\left(\left.\Delta_{p}\right|_{\operatorname{ker}\left(\Delta_{p}\right)^{\perp}}\right)^{-1} \delta d$, then we have $\sigma_{F}=P$. Since $F \phi_{k}=\phi_{k}$ we conclude that $\left\langle\phi_{k}, A \phi_{k}\right\rangle=\left\langle\phi_{k}, F A F \phi_{k}\right\rangle$. Hence, we may assume without loss of generality that $A=F A F$ and hence, $\sigma_{A}=P \sigma_{A} P$. Again we have the heat asymptotics

$$
\operatorname{Tr}\left(A e^{-\Delta_{p} t}\right) \sim C(p, n) \omega_{\operatorname{tr}}\left(\sigma_{A}\right) t^{-n / 2},
$$

and from Lemma A.1 $\omega_{t}\left(\left|\sigma_{A_{T}}\right|^{2}\right) \rightarrow 0$ as $T \rightarrow \infty$. Together these statements with the Karamata's Tauberian theorem imply Quantum ergodicity in the stated form exactly in the same way as in the proof of theorem 4.4.

In the above proof it was necessary to exclude the case $p=\frac{n-1}{2}$ because in this case the representation of $S O(n-1)$ on $\Lambda^{p} \mathbb{C}^{n-1}$ is not irreducible but splits into a direct sum of two irreducible representations. One reason for this is the existence of an involution defined by the Hodge star operator which commutes with the $S O(n-1)$ action. This actually causes the state $\omega_{t}$ to be non-ergodic in case $p=\frac{n-1}{2}$. To see this let $P_{ \pm} \in C\left(T_{1}^{*} X, \pi^{*} \operatorname{End}\left(\Lambda^{p} X\right)\right)$ defined by

$$
P_{ \pm}(\xi) v:=\frac{1}{2}\left(1 \pm \mathrm{i}^{p} i(\xi) *\right) i(\xi) \xi \wedge v
$$

where $*: \Lambda_{\mathbb{C}}^{p-1} X \rightarrow \Lambda_{\mathbb{C}}^{p+1} X$ is the Hodge star operator. Then $P_{ \pm}$ are orthogonal projections $C\left(T_{1}^{*} X, \pi^{*} \operatorname{End}\left(\Lambda_{\mathbb{C}}^{p} X\right)\right)$ which commute in addition with $P$. Therefore, we have $P=P_{+}+P_{-}$and

$$
\begin{aligned}
& \omega_{t}=\frac{1}{2}\left(\omega_{+}+\omega_{-}\right), \\
& \omega_{+}(a)=2 \omega_{t}\left(P_{+} a\right), \\
& \omega_{+}(a)=2 \omega_{t}\left(P_{-} a\right) .
\end{aligned}
$$

Again these states are invariant. The same proof as for theorem 5.1 now gives

Theorem 5.3. Suppose that $n>1$ is odd. Let $p=\frac{n-1}{2}$. If the $(n-1)$ frame flow is ergodic then $\omega_{ \pm}$are ergodic states with respect to $\beta_{t}$ on $C\left(T_{1}^{*} X, \pi^{*} \Lambda_{\mathbb{C}}^{p} X\right)$ and the systems $\left(C\left(T_{1}^{*} X, \pi^{*} \Lambda_{\mathbb{C}}^{p} X\right), \omega_{ \pm}\right)$are $\beta_{t}$-abelian.

It is amusing that also in case $p=\frac{n-1}{2}$ the non-ergodicity of the state $\omega_{t}$ is related to the existence of a "quantum symmetry", i.e. of a pseudodifferential operator which commutes with $\Delta_{p}$ and leaves the kernel of $\overline{\operatorname{Rg}(\delta)}$ invariant. Namely, for $p=\frac{n-1}{2}$ the operator $\mathrm{i}^{p+1} \Delta_{p}^{-1 / 2} \delta *$ is a selfadjoint involution on $\overline{\operatorname{Rg}(\delta)}$ whose principal symbol is precisely 
i ${ }^{p} i(\xi) *$. Hence, $P_{ \pm}$are the principal symbols of the projections to the \pm 1 eigenspaces of this involution. The proof of theorem 5.2 gives

Theorem 5.4. Suppose that $n$ is odd and $2 p=n-1$. Suppose that $\phi_{k}$ is an orthonormal sequence of eigen-p-forms satisfying

$$
\begin{aligned}
\Delta_{p} \phi_{k} & =\lambda_{k} \phi_{k}, \\
\delta \phi_{k} & =0, \\
\mathrm{i}^{p+1} \delta * \phi_{k} & = \pm \sqrt{\lambda_{k}} \phi_{k}
\end{aligned}
$$

such that the $\phi_{k}$ span $\overline{\operatorname{Ran}\left(\delta \pm \mathrm{i}^{p+1} \Delta_{p}^{-1 / 2} \delta * \delta\right)}$ and with $\lambda_{k} \nearrow \infty$. Then, if the $(n-1)$-frame flow is ergodic, the system is quantum ergodic in the sense that

$$
\lim _{N \rightarrow \infty} \frac{1}{N} \sum_{k \leq N}\left|\left\langle\phi_{k}, A \phi_{k}\right\rangle-\omega_{ \pm}\left(\sigma_{A}(\xi)\right)\right|=0,
$$

for all $A \in \Psi \mathrm{DO}_{c l}^{0}\left(X ; \Lambda_{\mathbb{C}}^{p} X\right)$. In particular there is a density one subsequence $\phi_{k}^{\prime}$ such that

$$
\lim _{k \rightarrow \infty}\left\langle\phi_{k}^{\prime}, A \phi_{k}^{\prime}\right\rangle=\omega_{ \pm}(\sigma(A)), \quad \text { for all } A \in \Psi \operatorname{DO}_{c l}^{0}\left(X ; \Lambda_{\mathbb{C}}^{p} X\right) .
$$

\section{Appendix A. ERgodicity of States For noncommutative CLASSICAL SYSTEMS}

Let $\mathcal{A}$ be a unital $C^{*}$-algebra. A state $\omega$ over $\mathcal{A}$ is a positive linear functional $\omega: \mathcal{A} \rightarrow \mathbb{C}$ with $\omega(1)=1$. The set of states $E_{\mathcal{A}}$ of $\mathcal{A}$ is a convex weakly-*-compact subset of $\mathcal{A}^{*}$ and its extreme points are the pure states $P_{\mathcal{A}}$. Each state $\omega$ gives via the GNS-construction rise to a representation $\pi_{\omega}: \mathcal{A} \rightarrow \mathcal{L}\left(\mathcal{H}_{\omega}\right)$ with a cyclic vector $\Omega_{\omega}$ such that $\omega(a)=\left\langle\Omega_{\omega}, \pi_{\omega}(a) \Omega_{\omega}\right\rangle$. Up to equivalence the triple $\left(\pi_{\omega}, \mathcal{H}_{\omega}, \Omega_{\omega}\right)$ is uniquely determined by its properties and we refer to it as the GNS triple.

In the following let $\alpha_{t}$ be a strongly continuous group of $*$-automorphisms of $\mathcal{A}$. The set of invariant states $E_{\mathcal{A}}^{\alpha_{t}}$ is again a weakly-*compact subset of $\mathcal{A}^{*}$. The extreme points in $E_{\mathcal{A}}^{\alpha_{t}}$ are called ergodic states. Hence, an invariant state $\omega$ is ergodic if it cannot be written as a convex linear combination of two other invariant states. If $\omega$ is an invariant state the group $\alpha_{t}$ can be uniquely implemented by a strongly continuous unitary group $U(t)$ on the GNS-Hilbert space $\mathcal{H}_{\omega}$ such that $U(t) \Omega_{\omega}=\Omega_{\omega}$ and $U(t)^{*} \pi_{\omega}(a) U(t)=\pi_{\omega}\left(\alpha_{t}(a)\right)$. Let $E_{\omega}$ be the orthogonal projection onto the space of $\alpha_{t}$-invariant vectors in $\mathcal{H}_{\omega}$. Then the pair $(\mathcal{A}, \omega)$ is called $\mathbb{R}$-abelian if all operators in $E_{\omega} \pi_{\omega}(\mathcal{A}) E_{\omega}$ commute pairwise. If we look at the following conditions 
(1) $E_{\omega}$ has rank one,

(2) $\omega$ is ergodic for $\alpha_{t}$, i.e. $\omega \in \mathcal{E}\left(E_{\mathcal{A}}^{\alpha_{t}}\right)$,

(3) $\left\{\pi_{\omega}(\mathcal{A}) \cup U(t)\right\}$ is irreducible on $\mathcal{H}_{\omega}$,

then it is known that $(1) \Rightarrow(2) \Leftrightarrow(3)$. If, moreover, either $(\mathcal{A}, \omega)$ is $\mathbb{R}$-abelian, or $\Omega_{\omega}$ is separating for $\pi_{\omega}(\mathcal{A})^{\prime \prime}$, then all three conditions are equivalent (see [BR79], Prop. 4.3.7, Th. 4.3.17, Th 4.3.20).

Now let $E \rightarrow X$ be a hermitian complex vector bundle over a compact Hausdorff space $X$. Then $\mathcal{A}=C(X$; $\operatorname{End}(E))$ is a unital $C^{*}$-algebra. Suppose that $\mu$ is some finite Borel measure with $\mu(X)=1$ and let $P \in C(X ; \operatorname{End}(E))$ be a non-trivial orthogonal projection onto a subbundle, i.e. $P$ has constant rank $k$. Then $\omega(a):=$ $\frac{1}{k} \int_{X} \operatorname{Tr}(P(x) a(x) P(x)) d \mu(x)$ is a state over $\mathcal{A}$. The GNS triple can be calculated explicitly and is given by $\mathcal{H}_{\omega}=L^{2}(X ; \operatorname{End}(E)) P$ with scalar product $\langle a, b\rangle=\frac{1}{k} \int_{X} \operatorname{Tr}\left(a^{*}(x) b(x)\right) d \mu(x)$ and $\Omega_{\omega}=P$. The action of $\mathcal{A}$ on $\mathcal{H}_{\omega}$ is by multiplication from the left. The von Neumann closure of $\pi_{\omega}(\mathcal{A})$ is given by $\pi_{\omega}(\mathcal{A})^{\prime \prime}=L^{\infty}(X ; \operatorname{End}(E))$. The commutant $\pi_{\omega}(\mathcal{A})^{\prime}$ of $\pi_{\omega}(\mathcal{A})$ can be identified with the opposite algebra of $P L^{\infty}(X ; \operatorname{End}(E)) P$ which acts on $L^{2}(X ; \operatorname{End}(E)) P$ by right multiplication. Note that $\Omega_{\omega}$ is separating for $\pi_{\omega}(\mathcal{A})^{\prime \prime}$ iff $P=\operatorname{Id}$. Now any continuous geometric flow on $E$ determines a continuous geometric flow on $\operatorname{End}(E)$. If the hermitian structure is preserved by the flow, this gives rise to a strongly continuous 1-parameter group $\alpha_{t}$ on $\mathcal{A}$. If $P$ and $\mu$ are invariant under the flow, then $\omega$ is an invariant state. If all invariant vectors in $L^{2}(X ; \operatorname{End}(E)) P$ are of the form $c P$ with $c \in \mathbb{C}$, then by the above $\omega$ is ergodic. In this case $E_{\omega} \pi_{\omega}(\mathcal{A}) E_{\omega}$ is just a multiplication by a number and the system is $\mathbb{R}$-abelian. If a state $\omega^{\prime}$ is majorized by $\omega$ then there exists a positive element $A \in P L^{\infty}(X ; \operatorname{End}(E)) P$ such that such that $\omega^{\prime}(a)=\omega(a A)$. Hence, if $P$ is up to a constant the only invariant element in $P L^{\infty}(X ; \operatorname{End}(E)) P$ then $\omega$ is ergodic.

Now denote by $\omega^{\prime}$ the restriction of the state $\omega$ to the subalgebra $\mathcal{B}=$ $P C(X ; \operatorname{End}(E)) P$. If $E^{\prime}$ denotes the subbundle onto which $P$ projects then clearly $\mathcal{B}=C\left(X ; \operatorname{End}\left(E^{\prime}\right)\right)$ and $\omega^{\prime}$ becomes the tracial state on $\mathcal{B}$. Ergodicity of the state is equivalent to the condition that all invariant elements in $P L^{\infty}(X ; \operatorname{End}(E)) P=L^{\infty}\left(X ; \operatorname{End}\left(E^{\prime}\right)\right)$ are multiples of $P$. Therefore, ergodicity of $\omega^{\prime}$ is equivalent to the ergodicity of $\omega$. Since $\Omega_{\omega^{\prime}}$ is separating for $\pi_{\omega^{\prime}}^{\prime \prime}(\mathcal{B})$ ergodicity of $\omega$ is equivalent to $E_{\omega^{\prime}}$ having rank one. As a consequence we get

Lemma A.1. If $A \in P L^{\infty}(X, E) P$ such that $\omega(A)=0$, then ergodicity of $\omega$ implies that

$$
\lim _{T \rightarrow \infty} \omega\left(\left|A_{T}\right|^{2}\right)=0
$$


where

$$
A_{T}=\frac{1}{T} \int_{0}^{T} \alpha_{t}(A) d t
$$

Proof. Since $A=P A P$ we also have $A_{T}^{2}=P A_{T}^{2} P$. By the above $E_{\omega^{\prime}}$ has rank one and its range is spanned by $P$. Now by the von Neumann ergodic theorem $A_{T}$ converges to $E_{\omega^{\prime}} A$ in $P L^{2}(X, E) P$. But since $\omega(A)=0$ we have $E_{\omega^{\prime}} A=0$ and therefore $A_{T}$ converges to 0 in $P L^{2}(X, E) P$. By definition this means that $\omega\left(\left|A_{T}\right|^{2}\right)$ converges to 0 .

Acknowledgements. The authors would like to thank J. Bolte for bringing the problem to their attention and for many useful discussions. The authors would also like to thank M. Brin, D. Dolgopyat, P. Gerard, N. Kamran, M. Lesch, W. Müller, M. Pollicott, I. Polterovich, P. Sarnak, A. Shnirelman, R. Schubert, J. Stix, J. Toth and S. Zelditch for useful discussions. The auhtors would also like to thank the anonymous referee whose comments helped improve the presentation of the results.

The first author would like to thank the Department of Theoretical Physics at the university of Ulm for their hospitality during his visit. This paper was started while the first author visited Max Planck Institute for Mathematics in Bonn, and completed while he was visiting IHES; their hospitality is greatly appreciated. The second author would like to thank McGill university and CRM Analysis laboratory for the hospitality during his Montreal visit.

\section{REFERENCES}

[Arn61] V. I. Arnold. Some remarks on flows of line elements and frames. Dokl. Akad. Nauk SSSR 138 (1961), 255-257.

[BGV92] N. Berline, E. Getzler, and M. Vergne. Heat kernels and Dirac operators, volume 298 of Grundlehren der Mathematischen Wissenschaften [Fundamental Principles of Mathematical Sciences]. Springer-Verlag, Berlin, 1992.

[BoK98] J. Bolte and S. Keppeler. Semiclassical time evolution and trace formula for relativistic spin-1/2 particles. Phys. Rev. Lett. 81 (1998), no. 10, 19871991.

[BoK99] J. Bolte and S. Keppeler. A semiclassical approach to the Dirac equation. Ann. Physics 274 (1999), no. 1, 125-162.

[Bol01] J. Bolte. Semiclassical expectation values for relativistic particles with spin 1/2. Invited papers dedicated to Martin C. Gutzwiller, Part III. Found. Phys. 31 (2001), no. 2, 423-444.

[BoG04] J. Bolte and R. Glaser. Zitterbewegung and semiclassical observables for the Dirac equation. J. Phys. A 37 (2004), no. 24, 6359-6373. 
[BoG04.2] J. Bolte, R. Glaser. A semiclassical Egorov theorem and quantum ergodicity for matrix valued operators. Comm. Math. Phys. 247 (2004), no. 2, 391-419.

[BR79] O. Bratteli and D. W. Robinson. Operator algebras and quantum statistical mechanics. Vol. 1. Springer-Verlag, New York, 1979. $C^{*}$ - and $W^{*}$ algebras, algebras, symmetry groups, decomposition of states, Texts and Monographs in Physics.

[Br75] M. Brin. Topological transitivity of one class of dynamical systems and flows of frames on manifolds of negative curvature. Funct. Anal. Appl. 9, 8-16 (1975)

[Br76] M. Brin. The topology of group extensions of Anosov systems. Math. Notes 18, 858-864, (1976)

[Br82] M. Brin. Ergodic theory of frame flows. In Ergodic Theory and Dynamical Systems II, Proc. Spec. Year, Maryland 1979-80, Progr. Math. 21, 163183, Birkhäuser, Boston, 1982.

[BrG80] M. Brin and M. Gromov. On the ergodicity of frame flows. Inv. Math. 60, 1-7 (1980)

[BrK84] M. Brin and H. Karcher. Frame flows on manifolds with pinched negative curvature. Comp. Math. 52, 275-297 (1984)

[BrP74] M. I. Brin and Ja. B. Pesin. Partially hyperbolic dynamical systems. Izv. Akad. Nauk SSSR Ser. Mat. 38 (1974), 170-212.

[BuP03] K. Burns and M. Pollicott. Stable ergodicity and frame flows. Geom. Dedicata, 98, 189-210 (2003)

[CV85] Y. Colin de Verdière. Ergodicité et fonctions propres du laplacien. Comm. Math. Phys. 102, 497-502, (1985)

[D82] N. Dencker. On the propagation of polarization sets for systems of real principal type. J. Funct. Anal. 46:351-372, 1982.

[DG75] J. J. Duistermaat and V. W. Guillemin. The spectrum of positive elliptic operators and periodic bicharacteristics. Invent. Math., 29(1), 39-79, (1975)

[DH72] J. J. Duistermaat and L. Hörmander. Fourier integral operators. II. Acta Mathematica, 128, 183-269, (1972)

[EW96] G. Emmrich and A. Weinstein. Geometry of the transport equation in multicomponent WKB approximations. Comm. Math. Phys. 176 (1996), no. $3,701-711$.

[Fri] T. Friedrich. Dirac operators in Riemannian geometry, volume 25 of Graduate Studies in Mathematics. American Mathematical Society. Translated from the 1997 German original by Andreas Nestke.

[FuHa91] W. Fulton and J. Harris. Representation theory, volume 129 of Graduate Texts in Mathematics. Springer Verlag, New York, 1991.

[GrSee95] G. Grubb and R. Seeley. Weakly parametric pseudodifferential operators and Atiyah-Patodi-Singer boundary problems. Invent. Math. 121, 481$529,(1995)$

[GMMP97] P. Gérard, P. Markowich, N. Mauser and F. Poupaud. Homogenization limits and Wigner transforms. Comm. Pure Appl. Math. 50 (1997), no. 4, 323-379. Erratum: Comm. Pure Appl. Math. 53 (2000), no. 2, 280-281.

[LM89] H. B. Lawson, Jr. and M. Michelsohn. Spin geometry, volume 38 of Princeton Mathematical Series. Princeton University Press, Princeton, NJ, 1989. 
[San99] M. R. Sandoval. Wave-trace asymptotics for operators of Dirac type. Comm. PDE 24 (1999), no. 9-10, 1903-1944.

[See67] R. T. Seeley. Complex powers of an elliptic operator. In Singular Integrals (Proc. Sympos. Pure Math., Chicago, Ill., 1966), pages 288-307. Amer. Math. Soc., Providence, R.I., 1967.

[Shn74] A. I. Shnirelman. Ergodic properties of eigenfunctions. (Russian) . Uspehi Mat. Nauk 29, 181-182, (1974).

[Shn93] A. I. Shnirelman. On the asymptotic properties of eigenfunctions in the regions of chaotic motion. In V. Lazutkin KAM theory and semiclassical approximations to eigenfunctions. Ergebnisse der Mathematik und ihrer Grenzgebiete (3), 24. Springer-Verlag, Berlin, 1993.

[Tay81] M. E. Taylor. Pseudodifferential operators, volume 34 of Princeton Mathematical Series. Princeton University Press, Princeton, N.J., 1981.

[Zel87] S. Zelditch. Uniform distribution of eigenfunctions on compact hyperbolic surfaces. Duke Math. J. 55, 919-941 (1987)

[Zel96] S. Zelditch. Quantum ergodicity of $C^{*}$ dynamical systems. Comm. Math. Phys. 177, 507-528, (1996)

Department of Mathematics and Statistics, McGill University, 805 Sherbrooke Str. West, Montréal QC H3A 2K6, Canada.

E-mail address: jakobson@math.mcgill.ca

Mathematisches Institut, Universität Bonn, Beringstrasse 1, D-53115 Bonn, Germany

E-mail address: strohmai@math.uni-bonn.de 